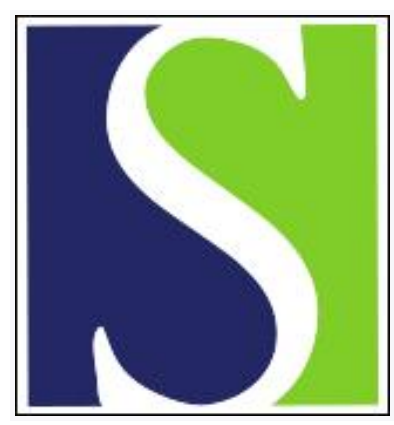

Scand J Work Environ Health 1997;23(3):161-163

https://doi.org/10.5271/sjweh.194

Issue date: Jun 1997

Evidence-based intervention for musculoskeletal disorders

by Malmivaara $\mathrm{A}$

This article in PubMed: www.ncbi.nlm.nih.gov/pubmed/9243725

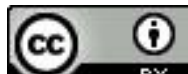




\section{Evidence-based intervention for musculoskeletal disorders}

A well-designed randomized controlled trial is the most valid method for obtaining evidence on the effectiveness of a particular intervention (1). During recent years several systematic reviews and national guidelines based on randomized controlled trials have been published on the effectiveness of intervention for musculoskeletal disorders (2-8). This issue of the Scandinavian Journal of Work, Environment \& Health provides a systematic review especially relevant for occupational health care personnel. It concerns the effectiveness of intervention on vocational outcomes of low-back pain (9).

The concept of random allocation as an important aspect of the design of scientific experiments was first proposed by Fisher in 1935 (10). The first controlled trial with a properly randomized reference group was for streptomycin in the treatment of tuberculosis in 1948. The study showed significantly better patient survival and radiological improvement for streptomycin treatment. Since the 1950s there has been a vast number of clinical trials, mainly with drug therapy. After the thalidomide disaster in the early 1960s, official approval became a requirement for clinical trials including drugs or for the placement of drugs on the market.

In his widely referred to book Effectiveness and Efficiency, published in the 1970s, Archie Cochrane proposed that other forms of health care intervention than drug therapy should also be subjected to similar rigorous assessment of effectiveness in randomized controlled trials (11). In 1992 the first Cochrane Collaboration Centre was founded in Oxford. By now several Cochrane centers have been established around the world to gather, synthesize, and disseminate information on effective health care intervention in various fields of medicine.

The justification for randomized controlled trials is based on their capability to eliminate bias (10). The uncontrolled trials have the potential to provide a very distorted view of intervention when the natural course of the condition is not controlled. These before-after comparisons usually lead to overenthusiastic recommendations in favor of the intervention when compared with the situation with properly controlled trials. Using historical controls also poses a threat to validity from two main sources, subject selection and experimental environment. Historical controls are hardly ever comparable with those of the intervention group, and statistical adjustment cannot control for all the potential confounders. The experimental environment differs also, and the quality of the recorded data for the historical controls is inferior. Thus validity problems arise from both the intrinsic and extrinsic determinants of outcome. Consequently, studies employing historical controls usually tend to exaggerate the value of an intervention. Problems with concurrent nonrandomized controls arise from the lack of concealment of treatment allocation, when the investigator is aware in advance which intervention will be given to the particular subject or group of subjects. This situation usually leads to poorer comparability for the explicit base-line characteristics and unsolved differences in the hidden characteristics.

Although randomization is an essential feature of a scientific intervention study, its acceptance is only a starting point for a rigorously planned and executed randomized controlled trial. In planning these studies, one can make use of standardized criteria for the methodological quality of randomized controlled trials $(1,4,9,12)$.

The ethical requirements for randomized controlled trials are stated in the Declaration of Helsinki, first issued by the World Medical Association in 1960. The declaration states that, to be considered ethical, randomized controlled trials must meet high scientific and organizational standards. Ethical committees should also take into account the scientific rigor of each study and its feasibility. Informed consent implies that the potential subjects for the trial must be adequately informed of the objectives, methods, anticipated benefits, and potential hazards of the study. In addition the subjects 
should be informed of their liberty to abstain from participation and that they are free to withdraw at any time.

Collective ethics is an important aspect that speaks strongly in favor of randomized controlled trials. These trials have an ability to promote medical progress effectively so that all patients benefit from superior treatment and other forms of intervention. In fact, when a clinical trial is planned, aspects concerning both individual ethics and collective ethics should be taken into account, and an appropriate balance between the two should be reached (10).

Reliable information from randomized controlled trials on the cost-effectiveness of medical intervention is scarce. High-quality randomized controlled trials incorporating economic analysis in their study design hardly exist in any field of medicine (13). Although the economic variables are time- and placedependent, it is valuable to assess both the use of health care resources and their monetary costs prospectively within the experimental setting (14). The resources in health care are limited and good cost-effectiveness data allow evidence-based decisions to be made on the wise use of these resources in promoting health for the population.

Concerning the calculation of indirect costs (ie, those due to sickness absence) a new method has recently been developed in health economics (15) in lieu of the human capital based analysis, which simply calculates the salary losses of workers. According to this new friction-cost analysis, the short sick leaves lead to actual losses in production and thus also to economic losses from the point of view of the whole economy. In cases of long absences or disability pensions, the employee may be replaced by another worker, and then there may not be any economic loss for society, although, for example, insurance companies pay for the worker. Friction-cost analysis is especially useful in cases of high unemployment, as is prevalent in Europe today.

As shown in the study by van der Weide and her colleagues (9), the method of analyzing papers in a systematic review is similar to that of any scientific study, in which each step is based on an a priori designed study protocol. The protocol determines the literature search, the inclusion criteria, the assessment of the validity of the individual reports and, finally, the method of assessing the overall level of evidence. This procedure can be used also in a cumulative systematic review in which the inferences are modified once new studies enter the material (16).

In systematic reviews it is mandatory to take the methodological rigor of the study design into account and draw conclusions primarily on the randomized controlled trials which rate best in terms of validity. Varying criteria have been developed for assessing the validity of individual trials. Differences exist in both the items included in the criteria, because of their assumed importance in terms of validity, and the weights placed on these items. Therefore the validity scores obtained by different criteria may vary considerably and consequently may lead to different inferences (17). One of the questions is whether the size of a study should be included as an item of methodological quality or not. Study size is, in fact, not a criterion of validity but one of precision. van der Weide and her colleagues (9) have cleverly solved the problem of how to include study size in their of evaluation. After the methodological quality was assessed, the studies considered adequate but having negative results were tested for power, and, if the power was inadequate, they were excluded from the review. Besides producing false negative results in individual studies, the studies with small numbers may hamper the validity of systematic reviews since the size of a study has a bearing on whether or not it will be published, and studies with small numbers thus contribute to publication bias.

One important item of validity in experimental studies is adherence (compliance) to study protocol (18). If, for example, the adherence to a particular type of intervention is very poor, the study will have no effect on the intervention. This result would certainly be biased, and this example illustrates that the actual degree of adherence is indeed a matter of validity.

One challenge is also how to incorporate knowledge of subject matter into systematic reviews in order to decrease the inherent reductionism of the analyses and maximize the gain from the vast amount of information included in the original studies. 
Systematic reviews do have a great impact both on the guidelines for practitioners with respect to the recommendable forms of intervention and on future randomized controlled trials. The latter form of impact is due to the importance of reviews in creating standards for the methodological quality of trials. Therefore systematic reviews may extend their influence far into the future.

Judgments on effective intervention should be made on the basis of the best scientific evidence available, and therefore constant effort is required to heighten the methodological rigor of both original studies and systematic reviews. For the latter, one can take advantage of the methods available for analyzing systematic reviews (ie, systematic reviews of the systematic reviews) (19).

The systematic review of van der Weide et al reveals that the scientific basis for the effectiveness of intervention on vocational outcomes of low-back pain is rather low. The evidence has earlier been found to be even poorer for other conditions (eg, neck pain) (4). The review points out that it is feasible to do randomized controlled trials relevant to occupational health care practice. As numerous important hypotheses and current practices in occupational health care can be tested by randomized controlled trials, it is a challenge for scientists working in the field to undertake these studies.

\section{References}

1. NHS Center for Reviews and Dissemination. Undertaking systematic reviews of research on effectiveness. York: NHS Center for Reviews and Dissemination, University of York, 1996. CRD report 4.

2. Bigos S, Bowyer O, Braen G, Brown K, Deyo R, Haldeman S, et al. Acute low back problems in adults. Rockville (MD): Agency for Health Care Policy and Research, Public Health Services, US Department of Health and Human Services, 1994. Clinical practice guideline, no 14.

3. Faas A, Chavannes AW, Koes BW, van den Hoogen JMM, Mens JMA, Smeele LJM, et al. Practice guideline 'low back pain'. Utrecht: Dutch College of General Practitioners, 1996.

4. Koes BW, Bouter LM, Beckerman H, van der Heijden GJMG, Knipschild PG. Physiotherapy exercises and back pain: a blinded review. BMJ 1991;302:1572-6.

5. Nachemson A. Ont i ryggen: orsaker, diagnostik och behandling [Back pain: causes, diagnosis, treatment]. Stockholm: Statens Beredning för Utvärdering av medicinsk metodik, 1991.

6. van Tulder MW, Koes BW, Bouter LM. Low back pain in primary care: effectiveness of diagnostic and therapentic interventions. Amsterdam: EMGO Institute, 1996.

7. Waddel G, Feder G, McIntosh, Lewis M, Hutchinson A. Low back pain evidence review. London: Royal College of General Practitioners, 1996

8. Finnish Academy. Selkäsairaudet konsensuskokous 14.-16.10. [Consensus meeting on low back disorders]. Helsinki: Suomen Akatemia, 1996. Suomen Akatemian julkaisuja 6/96.

9. van der Weide WE, Verbeek JHAM, van Tulder MW. Vocational outcome of intervention for low-back pain. Scand J Work Environ Health 1997;23(3):165-178.

10. Pocock SJ. Clinical trials: a practical approach. Chichester: John Wiley \& Sons, 1983.

11. Cochrane AL. Effectiveness and efficiency: random reflections on health services. Cambridge (England): Cambridge University Press, 1989.

12. Chalmers TC, Smith H, Blackburn B, Silverman B, Schroeder B, Reitman D, et al. A method for assessing the quality of a randomized control trial. Contr Clin Trials 1981;2:31-49.

13. Mason JM, Drummond MF. Cost-effectiveness registers for health technologies: never mind the width, feel the quality? Presented at the $10^{\text {th }}$ Annual Meeting of the International Society for Technology Assessment in Health Care, Baltimore (MD), June 19-22, 1994.

14. Drummond M, Davies L. Economic analysis alongside clinical trials. Int J Technol Assess Health Care 1991;7:561-73.

15. Koopmanschap MA, Rutten FFH, van Ineveld BM, van Roijen L. The friction cost method for measuring the indirect costs of disease. J Health Econ 1995;14:171-89.

16. Lau J, Antman EM, Jimenez-Silva J, Kupelnick B, Mosteller F, Chalmers TC. Cumulative systematic review of therapeutic trials for myocardial infarction. N Engl J Med 1992;327:248-54.

17. Moher D, Jadad A, Nichol G, Penman M, Tugwell P, Walsh S. Assessing the quality of RCTs: an annotated bibliography of scales and checklists. Contr Clin Trials 1995;16:62-73.

18. Miettinen OS. Theoretical epidemiology: principles of occurrence research in medicine. New York (NY): John Wiley \& Sons, 1985.

19. Sacks HS, Berrier J, Reitman D, Ancona-Berk VA, Chalmers TC. Systematic reviews of randomized controlled trials. N Engl J Med 1987;316:450_-5.

\author{
Antti Malmivaara, $M D$ \\ Department of Occupational Health \\ Finnish Institute of Occupational Health \\ Topeliuksenkatu 41 a $A$ \\ FIN-00250 Helsinki Finland
}

